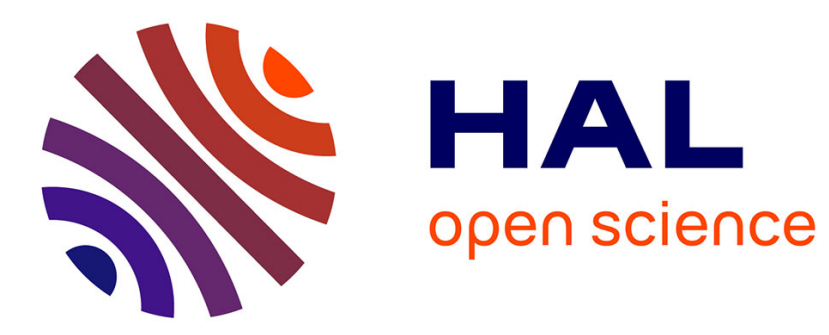

\title{
Multi-State Physics Model for the Reliability Assessment of a Component under Degradation Processes and Random Shocks
}

Yan-Hui Lin, Yanfu Li, Enrico Zio

\section{- To cite this version:}

Yan-Hui Lin, Yanfu Li, Enrico Zio. Multi-State Physics Model for the Reliability Assessment of a Component under Degradation Processes and Random Shocks. ESREL 2013, Sep 2013, Amsterdam,

Netherlands. pp.1-7. hal-00838745

\section{HAL Id: hal-00838745}

https://hal-centralesupelec.archives-ouvertes.fr/hal-00838745

Submitted on 26 Jun 2013

HAL is a multi-disciplinary open access archive for the deposit and dissemination of scientific research documents, whether they are published or not. The documents may come from teaching and research institutions in France or abroad, or from public or private research centers.
L'archive ouverte pluridisciplinaire HAL, est destinée au dépôt et à la diffusion de documents scientifiques de niveau recherche, publiés ou non, émanant des établissements d'enseignement et de recherche français ou étrangers, des laboratoires publics ou privés. 


\title{
Multi-State Physics Model for the Reliability Assessment of a Component under Degradation Processes and Random Shocks
}

\author{
Y.H. Lin \& Y.F. Li \\ Chair on Systems Science and the Energetic Challenge, European Foundation for New Energy - Electricité de \\ France, at École Centrale Paris - Supelec, France \\ E. Zio \\ Chair on Systems Science and the Energetic Challenge, European Foundation for New Energy - Electricité de \\ France, at École Centrale Paris - Supelec, France
}

Energy Department, Politecnico di Milano, Milano, Italy

\begin{abstract}
We extend a multi-state physics model (MSPM) framework for component reliability assessment by including semi-Markov and random shock processes. Dependences between the two processes are considered. A Monte Carlo simulation algorithm is developed to compute component reliability. An example is illustrated with respect to a literature case study.
\end{abstract}

\section{INTRODUCTION}

Failures of components generally occur in two modes: degradation failures due to physical deterioration in the form of wear, erosion, fatigue, etc, and catastrophic failures due to damages caused by sudden shocks in the form of jolts, blows, etc (Li \& Pham 2005, Wang et al. 2011). In the past decades, a number of degradation models have been proposed in the field of reliability engineering (Gebraeel et al. 2009, Lawless \& Crowder 2004, Elsayed \& Liao 2004, Lisnianski \& Levitin 2003, Giorgio et al. 2011, Kim \& Makis 2009, Li et al. 2012). They can be grouped into the following categories ( $\mathrm{Li}$ et al. 2012): statistical distributions (e.g. Bernstein distribution (Gebraeel et al. 2009)), stochastic processes (e.g. Brownian motion and Gamma process) (Lawless \& Crowder 2004, Elsayed \& Liao 2004), and multi-state models (Lisnianski \& Levitin 2003, Giorgio et al. 2011, Kim \& Makis 2009).

Most of the existing models are typically built upon degradation sample data from historical collection (Gebraeel et al. 2009, Elsayed \& Liao 2004, Giorgio et al. 2011) or expensive degradation tests (Lawless \& Crowder 2004). For critical components, it is often difficult or even impossible to collect degradation/failure samples, especially under operational conditions ( $\mathrm{Li}$ et al. 2012), so that the degradation models mentioned above might not be feasible for reliability assessment. An alternative is to resort to failure physics and structural reliability models, which do not require degradation/failure sample data and incorporate the knowledge of physics of failure (Kostandyan \& Sørensen 2012). Recently, Unwin et al. (2011) have proposed a multi-state physics model (MSPM) for modeling nuclear component degradation, also accounting for the effects of environmental factors (e.g. temperature and stress) within certain predetermined ranges (Fleming et al. 2010).

Random shocks also need to be accounted for because they can bring considerable variations to the environmental factors, even outside their predetermined ranges (Nakagawa 2007). In the literature, random shocks are typically modeled by Poisson processes (Li \& Pham 2005, Nakagawa 2007, Bai et al. 2006, Wang \& Pham 2012, Esary \& Marshall 1973), distinguishing two main types, extreme shock and cumulative shock processes (Bai et al. 2006), according to the severities of the damages. The former type could directly lead the component to immediate failure (Gut 1999, Anderson 1987), whereas the latter increases the degree of damage in a cumulative way (Agrafiotis \& Tsoukalas 1995, Nakagawa \& Kijima 1989).

The degradation processes subject to random shocks have been intensively studied (Li \& Pham 2005, Wang et al. 2011, Wang \& Pham 2012, Esary \& Marshall 1973, Ye et al. 2011, Fan et al. 2000, Klutke \& Yang 2002, Wortman et al. 1994). Esary \& Marshall (1973) have considered extreme shocks in the component reliability model, whereas Wang et al. (2011), Klutke \& Yang (2002), Wortman et al. (1994) have modeled the influences of cumulative shocks onto the degradation model. Both types of random shocks have been considered by (Li \& Pham 2005, Wang \& Pham 2012). Additionally, Ye et al. (2011), Fan et al. (2000) have considered that higher severity of degradation can lead to higher probability that a random shock causes extreme damage.

The contribution of this work is twofold: first, we extend our previous MSPM framework ( $\mathrm{Li}$ et al. 2012) to semi-Markov modeling, which is more general and describes the fact that the time transition to a state can depend on the residence time in the 
current state and hence is more suitable for considering maintenances (Huzurbazar \& Williams 2005); then, we integrate the random shock model into the MSPM framework. We propose a general random shock model, where the probability of a random shock resulting in extreme damage and cumulative damage are both dependent on the component degradation state and residence time in the state. The rest of this paper is organized as follows. Section 2 introduces the semi-Markov scheme into the MSPM framework. Section 3 presents the random shock model; in Section 4, this integration into MSPM is presented. Monte Carlo simulation procedures for component reliability assessment are presented in Section 5. Section 6 uses a numerical example regarding a case study of literature, to illustrate the proposed model. Section 7 concludes the work.

\section{MSPM OF COMPONENT DEGRADATION PROCESSES}

The following assumptions are made for the extended MSPM framework (Li et al. 2012) based on semiMarkov processes:

- The degradation process has a finite number of states $\mathbf{S}=\{0,1, \ldots, M\}$ where states ' $O$ ' and ' $M$ ' represent the complete failure state and perfect functioning state, respectively; the component is partially functioning in all generic intermediate degradation states $i(0<i<M)$, although partially.

- The degradation follows a continuous-time semi-Markov process; the transition rate between state $i$ and state $j$, denoted by $\lambda_{i, j}\left(\tau_{i}, \boldsymbol{\theta}\right)$, is a function of $\tau_{i}$, which is the residence time of the component being in the current state $i$ since the last transition, and $\boldsymbol{\theta}$, which represents the external influencing factors.

- The initial state (at time $t=0$ ) of the component is $M$.

- Maintenance can be carried out from any degradation state, except the complete failure state (in other words, there is no repair from failure).

Figure 1 presents the diagram of the semi-Markov component degradation process.

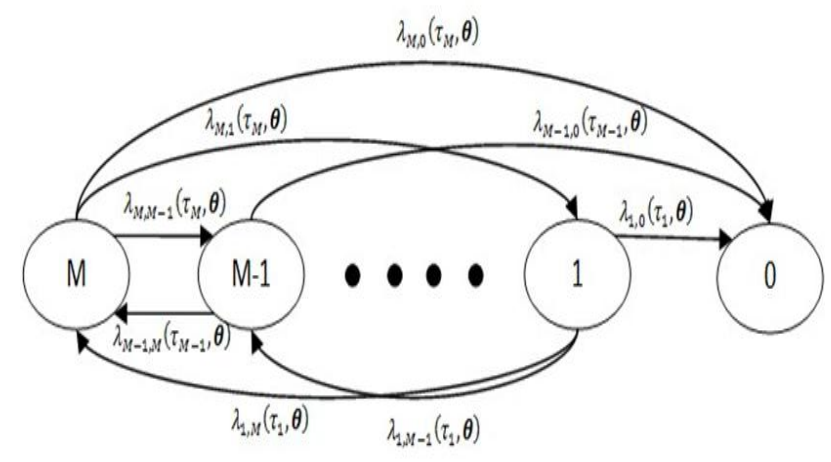

Figure 1. The diagram of the semi-Markov process.
The probability that the continuous time semiMarkov process will step to state $j$ at time $T_{n+1}$ in the next infinitesimal time interval $(t, t+\Delta t)$, given that it has arrived at state $i$ at time $T_{n}$ after $n$ transitions and remained stable in $i$ from $T_{n}$ until time $t$, is:

$$
\begin{aligned}
& \text { म } X_{n+1}=j, T_{n+1} \in[t, t+\Delta t] \mid\left\{X_{k}, T_{k}\right\}_{k=0}^{n-1}, \\
& \left.\left(X_{n}=i, T_{n}\right), T_{n} \leq t \leq T_{n+1}, \theta\right] \\
& =F X_{n+1}=j, T_{n+1} \in[t, t+\Delta t] \mid\left\{X_{k}, T_{k}\right\}_{k=0}^{n-1}, \\
& \left.T_{n} \leq t \leq T_{n+1}, \theta\right] \\
& =\lambda_{i, j}\left(\tau_{i}=t-T_{n}, \theta\right) \Delta t, \forall i, j \in \mathbf{S}, i \neq j
\end{aligned}
$$

where $X_{k}$ denotes the state of the component after $k$ transitions and $T_{k}$ denotes the time of arrival at $X_{k}$. The degradation transition rates can be obtained from the structural reliability analysis of the degradation processes (e.g. a crack propagation process, (Kostandyan \& Sørensen 2012, Fleming et al. 2010)), whereas the transition rates related to maintenance tasks can be estimated from the frequencies of maintenance activities.

The solution to the semi-Markov process model is the state probability vector $P(\mathrm{t})=\left\{p_{M}(t), p_{M}\right.$ $\left.{ }_{l}(t), \ldots, p_{0}(t)\right\}$ where $p_{i}(t)$ is the probability of the component being in state $i$ at time $t$. Since no maintenance is carried out from the component failure state and the component is regarded as functioning in all other intermediate alternative states, its reliability can be expressed as

$R(t)=1-p_{0}(t)$

where $p_{0}(t)$ is the probability of the complete failure state at time $t$.

Analytically solving the continuous time semiMarkov model with state residence time dependent transition rates is a difficult or sometimes impossible task, and the Monte Carlo simulation method is usually applied (Gillespie 1978, Rachelson et al. 2008).

\section{RANDOM SHOCKS}

The following assumptions are made on the random shock process:

- The random shocks are independent of the degradation process, but they can influence the degradation process (see Figure 2).

- The arrivals of random shocks follow a homogeneous Poisson process $\{N(t), t \geq 0\}$ (Bai et al. 2006) with constant arrival rate $\mu$, where the random variable $N(t)$ denotes the number of random shocks occurred until time $t$.

- The damages of random shocks are divided into two types: extreme and cumulative. 
- Extreme and cumulative shocks are mutually exclusive.

- The component fails immediately upon occurrence of extreme shocks.

- The probability of a random shock resulting in extreme damage is dependent on the current component degradation.

- The shock of cumulative damage can only influence the degradation transition departing from the current state and its impact on the degradation process is dependent on the current component degradation.

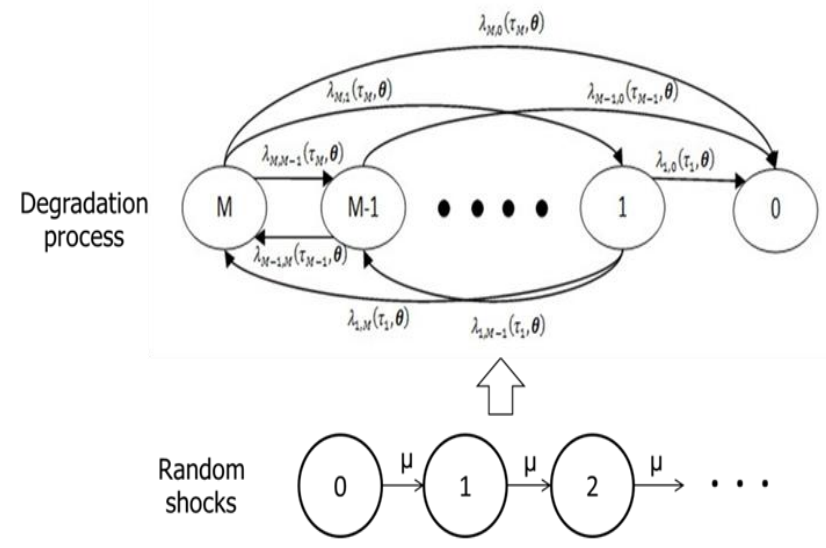

Figure 2. Degradation and random shock processes.

The first five assumptions are taken from (Wang \& Pham 2012). The sixth assumption reflects the aging effects addressed in (Fan et al. 2000) where the random shocks are more fatal to the component (i.e. more likely lead to extreme damages) when the component is in severe degradation states. However, the influences of cumulative shocks under aging effects have not been considered in Fan et al. (2000)'s model, as in the last assumption. In addition, the random shock damage is assumed to depend on the current degradation, characterized by three parameters: 1) the current degradation state $i, 2$ ) the number of cumulative shocks $m$ occurred while in the current degradation state since the last degradation state transition, 3) the residence time $\tau_{i, m}^{\prime}$ of the component in the current degradation state $i$ after $m$ cumulative shocks $\tau_{i, m}^{\prime} \geqslant 0$.

Let $\mathrm{p}_{(i, m)}\left(\tau_{i, m}^{\prime}\right)$ denote the probability that one shock results in extreme damage (the cumulative damage probability is then $1-\mathrm{p}_{(i, m)}\left(\tau_{i, m}^{\prime}\right)$ ). In case of cumulative shock, the degradation transition rates for the current state change at the moment of occurrence of shocks, whereas the other transition rates are not affected. Let $\lambda^{(m)}{ }_{i, j}\left(\tau^{\prime}{ }_{i, m}, \boldsymbol{\theta}\right)$ denote the transition rates after $m$ cumulative random shocks, where $\lambda^{(0)}{ }_{i, j}\left(\tau_{i, 0}{ }_{i, 0}, \boldsymbol{\theta}\right)$ holds the same expression as the transition rate $\lambda_{i, j}\left(\tau_{i, 0}, \boldsymbol{\theta}\right)$ in the pure degradation model, and the other transition rates (i.e. $m>0$ ) depend on the degradation and the external influencing factors, which are obtained from material science knowledge and data from shock tests (Chan \& Englert 2001). These quantities will be used as the key linking elements in the integration work of next section.

\section{INTEGRATION OF RANDOM SHOCKS IN THE MSPM}

Based on the first and second assumptions on random shocks, the new model that integrates random shocks into MSPM is shown in Figure 3.

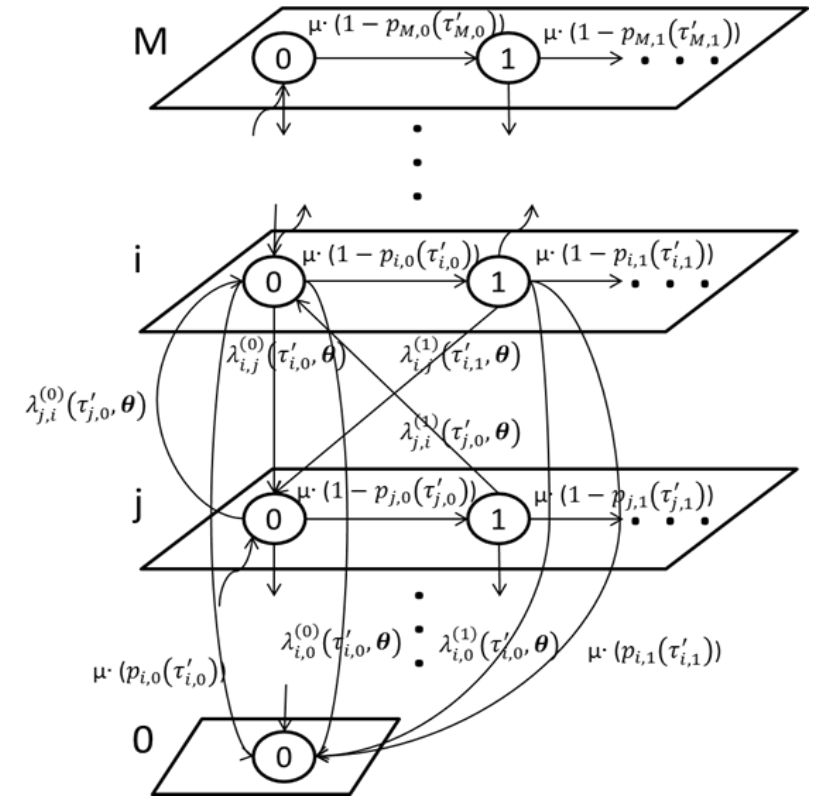

Figure 3. Degradation and random shocks processes.

In the model, the state of the component is represented by the pair $(i, m)$, where $i$ is the degradation state and $m$ is the number of cumulative shocks occurred during the residence time in the current state. For all the degradation states of component except for the state ' $O$ ', the number of cumulative shocks could range from $O$ to positive infinity. If the transition to a new degradation state occurs, the number of cumulative shocks is set to 0 , coherently with the last assumptions on random shocks. The state space of the new integrated model is denoted by $\mathbf{S}^{\prime}=$ $\{(M, 0),(M, 1),(M, 2), \ldots,(M-1,0),(M-1,1), \ldots$, $(0,0)\}$. The component is in failure whenever it reaches state $(0,0)$. The transition rate denoted by $\lambda_{(i, m),(j, n)}\left(\tau_{i, m}{ }_{i, m} \boldsymbol{\theta}\right)$ is residence time-dependent thus, rendering the process a continuous time semiMarkov process.

Suppose that the component is in a non-failure state $(i, m)$; then, we have three types of outgoing transition rates:

$$
\lambda_{i, m),(0,0)}\left(\tau_{i, m^{\prime}}^{\prime} \theta\right)=\mu \cdot\left(p_{i, m}\left(\tau_{i, m}^{\prime}\right)\right)
$$

the rate of occurrence of an extreme shock which will cause the component to go to state $(0,0)$,

$$
\lambda_{i, m),(i, m+1)}\left(\tau_{i, m}^{\prime}, \theta\right)=\mu \cdot\left(1-p_{i, m}\left(\tau_{i, m}^{\prime}\right)\right)
$$


the rate of occurrence of a cumulative shock which will cause the component to go to state $(i, m+1)$ and

$$
\lambda_{i, m),(j, 0)}\left(\tau_{i, m^{\prime}}^{\prime} \theta\right)=\lambda_{i, j}^{(m)}\left(\tau_{i, m^{\prime}}^{\prime} \theta\right)
$$

the rate of transition (i.e. degradation or maintenance) which will cause the component to go to state $(j, 0)$.

Similar to the solution of semi-Markov process presented in Section 2, the state probabilities of the new integrated model can be obtained by Monte Carlo simulation and the expression of reliability of component is:

$$
R(t)=1-p_{(0,0)}(t)
$$

\section{RELIABILITY ESTIMATION}

To generate the holding time $\tau_{i, m}{ }_{i, m}$ and the next state $(j, n)$ for the component arriving in any non-failure state $(i, m)$ at any time $t$, one proceeds as follows: two uniformly distributed random numbers $u_{1}$ and $u_{2}$ are sampled in the interval $[0,1]$; then, $\tau_{i, m}{ }^{\prime}$ is chosen so that

$\int_{0}^{\tau_{i}, m} \lambda_{i, m)}(s, \theta) d s=\ln \left(1 / u_{1}\right)$

where

$\lambda_{(i, m)}(s, \theta)=\sum_{\left(i^{\prime}, m^{\prime}\right)} \lambda_{(i, m),\left(i^{\prime}, m^{\prime}\right)}(s, \theta)$

and $(j, n)=\mathrm{a}^{*}$ that satisfies

$$
\begin{aligned}
& \sum_{k=0}^{a^{*}-1} \lambda_{i, m), k}\left(\tau_{i, m}^{\prime}, \theta\right)<u_{2} \lambda_{i, m)}\left(\tau_{i, m^{\prime}}^{\prime}, \theta\right)< \\
& \sum_{k=0}^{a^{*}} \lambda_{(i, m), k}\left(\tau_{i, m^{\prime}}^{\prime}, \theta\right)
\end{aligned}
$$

where $\mathrm{a}^{*}$ represents one state in the ordered sequence of all possible outgoing states of state $(i, m)$. The algorithm of Monte Carlo simulation for the reliability assessment of components under degradation and random shocks via MSMP, on a time horizon $\left[0, t_{\max }\right]$, is given by the following pseudo-code:

Set the maximum number of replications to $N_{\max }$

Set $k=0$

Set $k^{\prime}=0$

While $k<N_{\max }$

Initialize the system by setting $s=(M, 0)$ (initial state of perfect functioning), setting the time $t=0$ (initial time)

Set $t^{\prime}=0$

While $t<t_{\max }$

Sample a holding time $t$ ' by using equation

Sample an arrival state $(j, n)$ by using equation (8)

Set $t=t+t$,

Set $s=(j, n)$

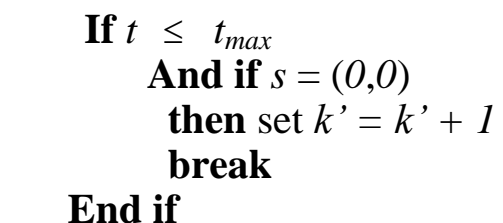

End While

Set $k=k+1$

End While $\square$

The estimated component reliability at time $t_{\max }$ can be obtained by

$\hat{\mathrm{R}}\left(t_{\text {max }}\right)=1-k^{\prime} / N_{\text {nax }}$

where $k^{\prime}$ represents the number of trials that end in the failure state, with a sample variance as follows (Lewis \& Böhm 1984)

$$
\left.\left.\operatorname{var}_{\hat{A_{\text {max }}}}=\hat{R} t_{\text {max }}\right)\left(1-\hat{R} t_{\text {max }}\right)\right) /\left(N_{\text {nax }}-1\right)
$$

\section{CASE STUDY AND RESULTS}

\subsection{Case study}

We illustrate the proposed modeling framework on a case study slightly modified from Fleming et al. (2010). The case study considers an Alloy 82/182 dissimilar metal weld in a primary coolant system of a nuclear power plant. The MSPM of the original crack growth is shown in Figure 4.

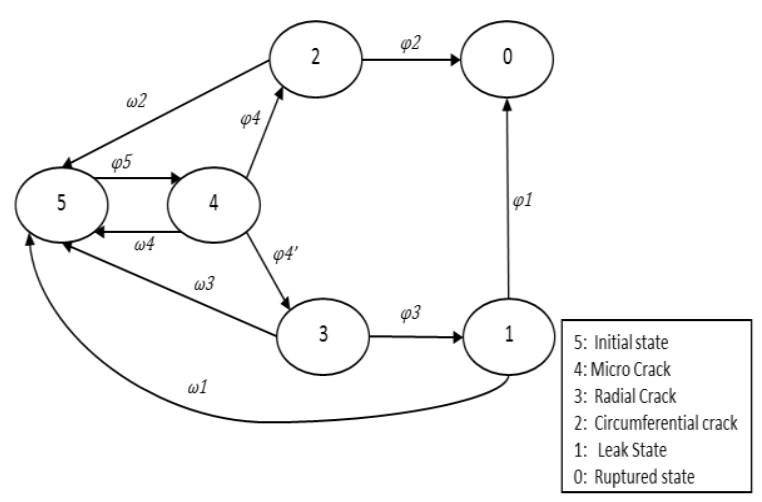

Figure 4. MSPM of crack development in Alloy 82/182 dissimilar metal welds.

In the Figure, $\varphi_{i}$ and $\omega_{i}$ represent the degradation transition rate and maintenance transition rate, respectively. Except for $\varphi_{5}, \varphi_{4}, \varphi_{4}$, and $\varphi_{3}$, all the other transition rates are assumed to be constant. The expressions of the transition rates and parameters of the model can be found in (Fleming et al. 2010).

For the random shocks, we assume the occurrence rate $\mu=1 / 15$, the same value in (Wang \& Pham 2012 ), and the probability of a random shock becoming extreme shock $\mathrm{p}_{(i, m)}\left(\tau^{\prime}{ }_{i, m}\right)=1-\exp [-\delta m(6-i)(2-$ $\left.\exp \left(-\tau^{\prime}{ }_{i, m}\right)\right]$, which takes the exponential formulation from Fan et al.'s work (Fan et al. 2000). In this formula, we use $m(6-i)\left(2-\exp \left(-\tau^{\prime}{ }_{i, m}\right)\right)$ to quantify the component degradation. It is noted that the quantity $2-\exp \left(-\tau_{i, m}^{\prime}\right)$ ranges from 1 to 2 , representing the relatively small effect of $\tau_{i, m}{ }_{i, m}$ onto the degradation, in 
comparison with the other two parameters $m$ and $i$, and $\delta$ is a predetermined constant which controls the influence of the degradation onto the probability $\mathrm{p}_{(i, m)}\left(\tau_{i, m}^{\prime}\right)$. In this study, we set $\delta=0.0001$. In addition, we assume the corresponding degradation transition rates after $m$ cumulative shocks to be $\lambda^{(m)}{ }_{i, j}\left(\tau_{i, m}{ }_{i, m}, \boldsymbol{\theta}\right)=(1+\varepsilon)^{\mathrm{m}} \lambda^{(m)}{ }_{i, j}\left(\tau^{\prime}{ }_{i, m}, \boldsymbol{\theta}\right)$, where $\varepsilon=0.3$ is the relative increment of transition rates after one cumulative shock happens, and the formulation $(1+\varepsilon)^{\mathrm{m}}$ is used to characterize the accumulated effect of such shocks.

\subsection{Results and analysis}

The Monte Carlo simulation over a time horizon of $t_{\max }=80$ years is run $N_{\max }=10^{6}$ times. The estimated component reliabilities with and without random shocks throughout the time horizon are shown in Figure 5, respectively.

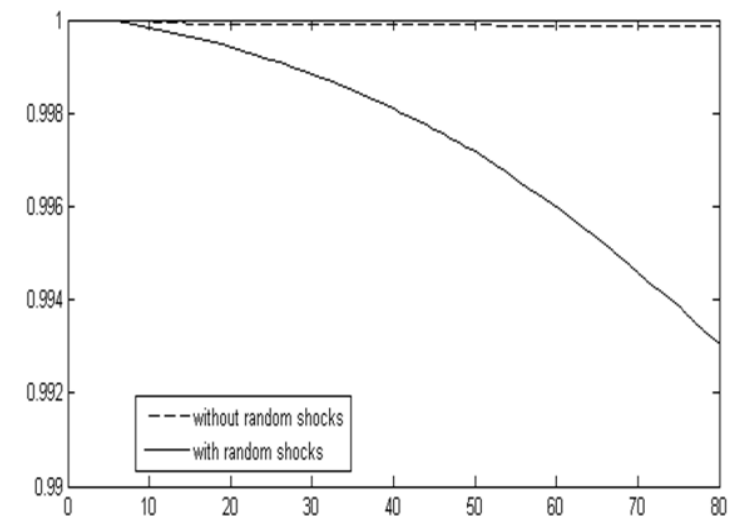

Figure 5. Reliability estimation of component with/without random shocks.

At year 80 , the estimated component reliability with random shocks is 0.9930, with sample variance equal to 6.95e-9. Compared with the case without random shocks (reliability equals to 0.9998 , with sample variance $2.00 \mathrm{e}-10$ ), the component reliability has decreased by 0.0068 . Table 1 presents the frequencies of different numbers of random shocks occurred per each trial of the simulation. The most likely number is around 5, which is consistent with our assumption on the occurrence rate $(\mu=1 / 15)$ of random shocks.

Table 1. Frequency of the number of random shocks occurred per trial (mission time $\mathrm{t}=80$ years)

\begin{tabular}{|c|c|}
\hline $\begin{array}{c}\text { Nb of random } \\
\text { shocks/trial }\end{array}$ & Percentage $(\%)$ \\
\hline 0 & 0.63 \\
\hline 1 & 3.14 \\
\hline 2 & 8.00 \\
\hline 3 & 13.55 \\
\hline 4 & 17.15 \\
\hline 5 & 17.56 \\
\hline
\end{tabular}

\begin{tabular}{|c|c|}
\hline 6 & 14.91 \\
\hline 7 & 10.83 \\
\hline 8 & 6.87 \\
\hline 9 & 3.90 \\
\hline$>9$ & 3.45 \\
\hline
\end{tabular}

In total, there are 6973 trials ended in failure, among which 4531 trials (64.98\%) are caused by extreme shocks. Table 2 records the number of trials and the number of trials ended with extreme shocks, as a function of numbers of cumulative shocks occurred per trial, respectively.

Table 2. Number of trials and number of trials ending with extreme shocks (mission time $\mathrm{t}=80$ years).

\begin{tabular}{|c|c|c|}
\hline $\begin{array}{c}\text { Nb of cumula- } \\
\text { tive shocks per } \\
\text { trial }\end{array}$ & Nb of trials & $\begin{array}{c}\text { Nb of trials } \\
\text { ending with ex- } \\
\text { treme shock }\end{array}$ \\
\hline 0 & 6345 & 0 \\
\hline 1 & 31739 & 367 \\
\hline 2 & 80292 & 633 \\
\hline 3 & 135676 & 812 \\
\hline 4 & 171526 & 809 \\
\hline 5 & 175569 & 743 \\
\hline 6 & 148844 & 500 \\
\hline 7 & 108101 & 332 \\
\hline 8 & 68579 & 172 \\
\hline 9 & 38964 & 90 \\
\hline 10 & 19569 & 43 \\
\hline 11 & 8998 & 19 \\
\hline 12 & 3683 & 11 \\
\hline$>12$ & 2115 & 0 \\
\hline
\end{tabular}

Based upon this Table, the influence of the number of cumulative shocks occurred per trial onto the probability of next random shock becoming extreme shock is shown by the curve in Figure 6 .

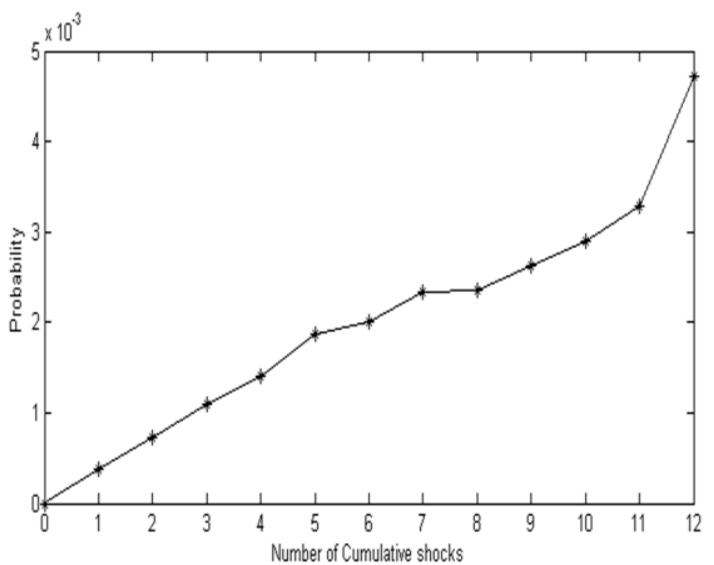

Figure 6. Probability of the next random shock resulting in extreme shock, given the number of cumulative shocks occurred per trial.

It is shown that high numbers of cumulative shocks lead to high probability of next random shock resulting in extreme shock. 
In order to characterize the influences of cumulative shocks to the degradation processes, we use the same case except requiring that the probability of a random shock becoming extreme shock is 0 , so that all the random shocks will be cumulative shocks. In Figure 7, we compare the estimated component reliability with cumulative shocks against the other two estimated probabilities in Figure 5. At year 80, the estimated component reliability with cumulative shocks is 0.9973 and the sample variance equals to $2.69 \mathrm{e}-9$. Due to the influence of cumulative shocks, the component reliability has decreased by 0.0026 .

By these results, it is seen that our model is able to characterize the influences of different types of random shocks onto the component reliability.

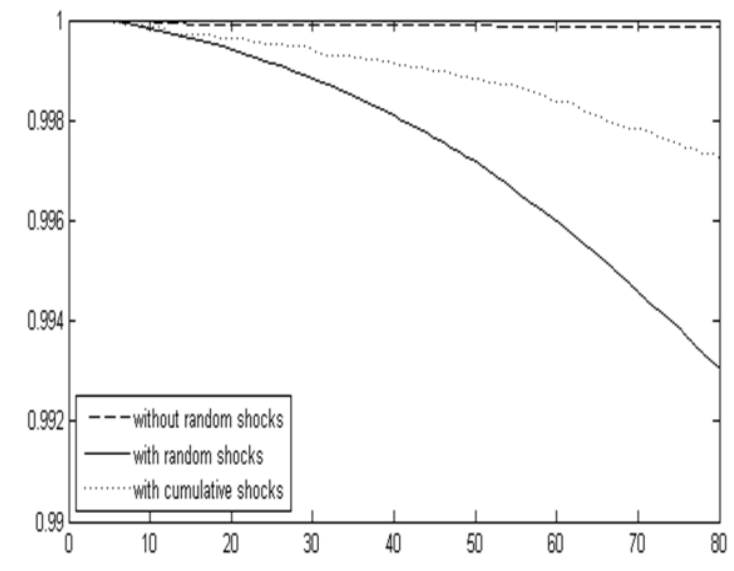

Figure 7. Component reliability functions with/without random shocks and with only cumulative shocks.

\section{CONCLUSION}

In this paper, a general degradation process dependent on random shocks has been proposed and integrated into a MSPM framework with semi-Markov processes for the reliability assessment of components. A literature case study has been considered to show the effectiveness and modeling capabilities of the proposal.

\section{REFERENCES}

Agrafiotis, G. \& Tsoukalas, M. 1995. On excess-time correlated cumulative processes. Journal of the Operational Research Society, 1269-1280.

Anderson, K. K. 1987. Limit theorems for general shock models with infinite mean intershock times. Journal of applied probability, 449-456.

Bai, J.-M., Li, Z.-H. \& Kong, X.-B. 2006. Generalized shock models based on a cluster point process. Reliability, IEEE Transactions on, 55, 542-550.

Chan, H. A. \& Englert, P. J. 2001. Accelerated stress testing handbook, JW.
Elsayed, E. \& Liao, H. 2004. A geometric Brownian motion model for field degradation data. International Journal of Materials and Product Technology, 20, 51-72.

Esary, J. \& Marshall, A. 1973. Shock models and wear processes. The annals of probability, 627-649.

Fan, J., Ghurye, S. \& Levine, R. A. 2000. Multicomponent lifetime distributions in the presence of ageing. Journal of applied probability, 37, 521-533.

Fleming, K. N., Unwin, S. D., Kelly, D., Lowry, P. P., Toloczko, M. B., Layton, R. F., Youngblood, R., Collins, D., Huzurbazar, A. V. \& Williams, B. 2010. Treatment of Passive Component Reliability in Risk-Informed Safety Margin Characterization.

Gebraeel, N., Elwany, A. \& Pan, J. 2009. Residual life predictions in the absence of prior degradation knowledge. Reliability, IEEE Transactions on, 58, 106-117.

Gillespie, D. T. 1978. Monte Carlo simulation of random walks with residence time dependent transition probability rates. Journal of Computational Physics, 28, 395-407.

Giorgio, M., Guida, M. \& Pulcini, G. 2011. An age-and statedependent Markov model for degradation processes. IIE Transactions, 43, 621-632.

Gut, A. 1999. Extreme shock models. Extremes, 2, 295-307.

Huzurbazar, A. V. \& Williams, B. 2005. Flowgraph models for complex multistate system reliability. Modern statistical and mathematical methods in reliability, 10, 247-262.

Kim, M. J. \& Makis, V. 2009. Optimal maintenance policy for a multi-state deteriorating system with two types of failures under general repair. Computers \& Industrial Engineering, 57, 298-303.

Klutke, G.-A. \& Yang, Y. 2002. The availability of inspected systems subject to shocks and graceful degradation. Reliability, IEEE Transactions on, 51, 371-374.

Kostandyan, E. E. \& Sørensen, J. D. 2012. Physics of failure as a basis for solder elements reliability assessment in wind turbines. Reliability Engineering \& System Safety.

Lawless, J. \& Crowder, M. 2004. Covariates and random effects in a gamma process model with application to degradation and failure. Lifetime Data Analysis, 10, 213227.

Lewis, E. \& Böhm, F. 1984. Monte Carlo simulation of Markov unreliability models. Nuclear Engineering and Design, 77, 49-62.

Li, W. \& Pham, H. 2005. Reliability modeling of multi-state degraded systems with multi-competing failures and random shocks. Reliability, IEEE Transactions on, 54, 297-303.

Li, Y.-F., Zio, E. \& Lin, Y.-H. 2012. A Multistate Physics Model of Component Degradation Based on Stochastic Petri Nets and Simulation. Reliability, IEEE Transactions on, 61, $921-931$

Lisnianski, A. \& Levitin, G. 2003. Multi-state system reliability: assessment, optimization and applications, World Scientific Publishing Company.

Nakagawa, T. 2007. Shock and damage models in reliability theory, Springer.

Nakagawa, T. \& Kijima, M. 1989. Replacement policies for a cumulative damage model with minimal repair at failure. Reliability, IEEE Transactions on, 38, 581-584.

Rachelson, E., Quesnel, G., Garcia, F. \& Fabiani, P. A simulation-based approach for solving generalized semimarkov decision processes. European Conference on Artificial Intelligence, 2008.

Unwin, S. D., Lowry, P. P., Layton, R. F., Heasler, P. G. \& Toloczko, M. B. 2011. Multi-state physics models of aging passive components in probabilistic risk assessment. Pacific Northwest National Laboratory (PNNL), Richland, WA (US).

Wang, Y. \& Pham, H. 2012. Modeling the dependent competing risks with multiple degradation processes and 
random shock using time-varying copulas. Reliability, IEEE Transactions on, 61, 13-22.

Wang, Z., Huang, H.-Z., Li, Y. \& Xiao, N.-C. 2011. An approach to reliability assessment under degradation and shock process. Reliability, IEEE Transactions on, 60, 852863.

Wortman, M., Klutke, G.-A. \& Ayhan, H. 1994. A maintenance strategy for systems subjected to deterioration governed by random shocks. Reliability, IEEE Transactions on, 43, 439445.

Ye, Z. S., Tang, L. C. \& Xu, H. Y. 2011. A distribution-based systems reliability model under extreme shocks and natural degradation. Reliability, IEEE Transactions on, 60, 246256. 